Bulletin of the Section of Logic

Volume 45:3/4 (2016), pp. 171-184

http://dx.doi.org/10.18778/0138-0680.45.3.4.03

Zofia Kostrzycka

\title{
INTERPOLATION IN NORMAL EXTENSIONS OF THE BROUWER LOGIC
}

\begin{abstract}
The Craig interpolation property and interpolation property for deducibility are considered for special kind of normal extensions of the Brouwer logic.

Keywords: normal extensions of the Brouwer logics, Kripke frames, interpolation property, amalgamation property
\end{abstract}

\section{Introduction}

In this paper we continue research on modal logics with and without the interpolation property within the family of normal extensions of Brouwer logic. The Brouwer logic is defined as follows: KTB $:=\mathbf{K} \oplus T \oplus B$ where:

$$
\begin{aligned}
T & :=\square p \rightarrow p \\
B & :=p \rightarrow \square \diamond p
\end{aligned}
$$

By a normal extension we mean a logic which is closed under the rules of modus ponens (MP), substitution and the Gödel rule of necessitation (RN). The Brouwer logic KTB is called to be non-transitive as it is characterized by the class of reflexive and symmetric (admitting non-transitive) frames.

In the paper [9] a class of logics without interpolation is described. The described logics are weakly transitive. In this paper we present some results for non-transitive logics determined by reflexive and symmetric Kripke frames being chains of points. So, we shall study the Brouwerian modal $\operatorname{logic} \mathbf{K T B} \cdot \mathbf{A l t}(\mathbf{3}):=\mathbf{K T B} \oplus$ alt $_{3}$ where

$$
\operatorname{alt}_{3}:=\square p \vee \square(p \rightarrow q) \vee \square((p \wedge q) \rightarrow r) \vee \square((p \wedge q \wedge r) \rightarrow s) .
$$


Let us emphasize that the logic KTB.Alt(3) is complete with respect to the class of reflexive and symmetric Kripke frames (possibly infinite) being chains of points.

THEOREM 1. [Byrd and Ullrich, 1977; Byrd, 1978] Every normal modal logic which is a proper extension of $\mathbf{K T B}$.Alt(3) has the finite model property and is finitely axiomatizable (and hence - decidable).

It is easily seen by the above theorem that the cardinality of the class $N E X T(\mathbf{K T B} \cdot \mathbf{A l t}(\mathbf{3}))$ is only countably infinite.

We may also consider logics determined by reflexive and symmetric Kripke frames with a larger degree of branching. The axiom $\left(\right.$ alt $\left._{3}\right)$ is a special case of more general axiom $\left(a l t_{n}\right), n \geq 3$ :

$$
\text { alt }_{n}:=\square p_{1} \vee \square\left(p_{1} \rightarrow p_{2}\right) \vee \ldots \vee \square\left(\left(p_{1} \wedge \ldots \wedge p_{n}\right) \rightarrow p_{n+1}\right) .
$$

In contrast to $N E X T(\mathbf{K T B} . \mathbf{A l t}(\mathbf{3}))$, the family of logics $N E X T(\mathbf{K T B} . \operatorname{Alt}(\mathbf{4})):=\mathbf{K T B} \oplus$ alt $_{4}$ is uncountably infinite, see [10].

\section{Preliminaries}

Let us recall some definitions. The symbol $\operatorname{Var}(\alpha)$ means the set of all propositional variables of the formula $\alpha$.

Definition 1. A logic L has the Craig interpolation property (CIP) if for every implication $\alpha \rightarrow \beta$ in $L$, there exists a formula $\gamma$ (interpolant for $\alpha \rightarrow \beta$ in $L)$ such that

$$
\alpha \rightarrow \gamma \in L \text { and } \gamma \rightarrow \beta \in L
$$

and $\operatorname{Var}(\gamma) \subseteq \operatorname{Var}(\alpha) \cap \operatorname{Var}(\beta)$.

The weaker notion of interpolation for deducibility is defined as follows:

DeFinition 2. A logic L has interpolation for deducibility (IPD) if for any $\alpha$ and $\beta$ the condition $\alpha \vdash_{L} \beta$ implies that there exists a formula $\gamma$ such that

$$
\alpha \vdash_{L} \gamma \text { and } \gamma \vdash_{L} \beta
$$

$\operatorname{Var}(\gamma) \subseteq \operatorname{Var}(\alpha) \cap \operatorname{Var}(\beta)$

It is a logical folklore that (CIP) together with (MP) and deduction theorem implies (IPD). It is also known that $\mathbf{K}, \mathbf{T}, \mathbf{K} \mathbf{4}$ and $\mathbf{S} \mathbf{4}$ have (CIP), 
see Gabbay [6]. Also the logics from $N E X T(\mathbf{S 4})$ are well characterized as regards interpolation (see [14], also [4], p.462-463). It is also known that S5 has (CIP). The last fact can be proven by applying a very general method of construction of inseparable tableaux (see i.e. [4], p. 446-449). The same method can be applied in the case of KTB. Therefore, without getting into details, we get:

Theorem 2. The logic KTB has (CIP).

The method of construction of inseparable tableaux is not applicable in the case of KTB.Alt(3) and its normal extensions. The following questions arise:

Question 1. Does the logic KTB.Alt(3) has (CIP) or (IDP)?

Question 2. Which logic from the family NEXT(KTB.Alt(3)) has (CIP) or (IDP)?

We shall answer question 1 in section 3 , whereas question 2 in section 4 . In the second case, our approach is purely semantic. We shall consider logics determined by class of Kripke frames $\mathcal{K}$. Formally, the logic determined by $\mathcal{K}$ is defined as follows:

$$
L(\mathcal{K}):=\{\alpha \in \text { Form }: \mathfrak{F} \models \alpha \text { for each } \mathfrak{F} \in \mathcal{K}\} .
$$

Note that the class $\mathcal{K}$ may consist of one frame only.

The properties (CIP) and (IPD) have an appropriate algebraic characterization, (see [14], [5]). The symbol $V(L)$ denotes the variety of algebras characterizing the logic $L$.

TheOREM 3. For any logic $L \in N E X T(\mathbf{K})$ the following are equivalent:

- $L$ possesses (CIP),

- $V(L)$ has the superamalgamation property.

TheOREM 4. For any logic $L \in N E X T(\mathbf{K})$ the following are equivalent:

- $L$ possesses (IPD),

- $V(L)$ has the amalgamation property.

By theory of duality between finite Kripke frames and finite modal algebras, the superamalgamation property and amalgamation property is 
transformed into appropriate properties (APK) and (SAPK) for class $\mathcal{K}$ of Kripke frames. We recall the notion of p-morphism first.

Definition 3. Let $\mathfrak{F}_{1}:=\left\langle W_{1}, R_{1}\right\rangle$ and $\mathfrak{F}_{2}:=\left\langle W_{2}, R_{2}\right\rangle$ be Kripke frames. A map $f: W_{1} \rightarrow W_{2}$ is a p-morphism from $\mathfrak{F}_{1}$ to $\mathfrak{F}_{2}$, if it satisfies the following conditions:

(p1) $f$ maps $W_{1}$ onto $W_{2}$,

(p2) for all $x, y \in W_{1}, x R_{1} y$ implies $f(x) R_{2} f(y)$,

(p3) for each $x \in W_{1}$ and for each $a \in W_{2}$, if $f(x) R_{2} a$ then there exists $y \in W_{1}$ such that $x R_{1} y$ and $f(y)=a$.

It is said that the frame $\mathfrak{F}_{1}$ is reducible to $\mathfrak{F}_{2}$ or that the frame $\mathfrak{F}_{2}$ is a p-morphic reduct of $\mathfrak{F}_{1}$.

A reduction $f$ of $\mathfrak{F}_{1}$ to $\mathfrak{F}_{2}$ is called a reduction of a model $\mathfrak{M}_{1}=\left\langle\mathfrak{F}_{1}, V_{1}\right\rangle$ to a model $\mathfrak{M}_{2}=\left\langle\mathfrak{F}_{2}, V_{2}\right\rangle$ if, for every variable $p$ and every point $x$ in $\mathfrak{F}$ :

$$
\left(\mathfrak{M}_{1}, x\right) \models p \quad \text { iff } \quad\left(\mathfrak{M}_{2}, f(x)\right) \models p .
$$

Second, we give the definitions of (APK) and (SAPK) for frames.

DeFinition 4. For any $\mathfrak{F}_{0}, \mathfrak{F}_{1}$ and $\mathfrak{F}_{2}$ in class $K$ and for any $p$-morphism $f_{1}: \mathfrak{F}_{1} \rightarrow \mathfrak{F}_{0}$ and $f_{2}: \mathfrak{F}_{2} \rightarrow \mathfrak{F}_{0}$ there exist $\mathfrak{F}$ in $K$ and $p$-morphisms $g_{1}: \mathfrak{F} \rightarrow \mathfrak{F}_{1}$ and $g_{2}: \mathfrak{F} \rightarrow \mathfrak{F}_{2}$ such that $f_{1} \circ g_{1}=f_{2} \circ g_{2}$ (see Figure 1 ).

Superamalgamation property for frames except (APK) requires the additional condition (SAPK):

$$
\forall_{x \in \mathfrak{F}_{1}} \forall_{y \in \mathfrak{F}_{2}}\left[f_{1}(x)=f_{2}(y) \Rightarrow \exists_{z \in \mathfrak{F}} g_{1}(z)=x \wedge g_{2}(z)=y\right] .
$$

$\mathfrak{F}_{1}$

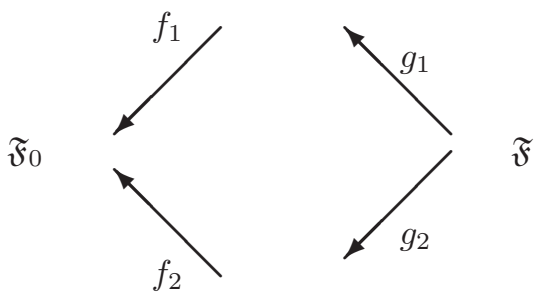

$\mathfrak{F}_{2}$

Figure 1. 


\section{Interpolation of KTB.Alt $(3)$}

In this section we shall prove that the logic KTB.Alt(3) does not have (CIP). We define the suitable formula $\alpha \rightarrow \beta$ as follows:

$$
\begin{aligned}
\alpha & :=\diamond(p \wedge q) \wedge \diamond(p \wedge \neg q), \\
\beta & :=[\diamond(\neg p \wedge r) \wedge \diamond(\neg p \wedge \neg r)] \rightarrow \perp .
\end{aligned}
$$

One may see that $\operatorname{Var}(\alpha) \cap \operatorname{Var}(\beta)=\{p\}$. First, we prove that

Lemma 1. The formula $\alpha \rightarrow \beta$ is a theorem of KTB.Alt $(\mathbf{3})$.

Proof: Suppose, on the contrary, that $\alpha \rightarrow \beta \notin$ KTB.Alt(3). There is some reflexive, symmetric and linear Kripke frame $\mathfrak{F}=\langle W, R\rangle$, a point $x_{0} \in W$ and a valuation $V$, such that $x_{0} \nvdash_{V} \alpha \rightarrow \beta$. Then

$$
\begin{aligned}
& x_{0} \models_{V} \alpha \\
& x_{0} \not \models_{V} \beta .
\end{aligned}
$$

From (3.1) and (3.2) we get:

$$
\begin{aligned}
& x_{0} \models_{V} \diamond(p \wedge q) \wedge \diamond(p \wedge \neg q), \\
& x_{0} \models_{V} \diamond(\neg p \wedge r) \wedge \diamond(\neg p \wedge \neg r) .
\end{aligned}
$$

Ten we get:

$$
\begin{aligned}
& x_{0} \models_{V} \diamond(p \wedge q), \\
& x_{0} \models_{V} \diamond(p \wedge \neg q), \\
& x_{0} \models_{V} \diamond(\neg p \wedge r), \\
& x_{0} \models_{V} \diamond(\neg p \wedge \neg r) .
\end{aligned}
$$

From (3.5)-(3.8) we conclude that there are four points $x_{i} \in W, i:=$ $1, \ldots, 4$ such that $x_{0} R x_{i}$, and:

$$
x_{1} \models_{V} p \wedge q, \quad x_{2} \models_{V} p \wedge \neg q, \quad x_{3} \models_{V} \neg p \wedge r, \quad x_{4} \models_{V} \neg p \wedge \neg r,
$$

and we conclude that $x_{i} \neq x_{j}$ if $i \neq j$ for $i, j:=1, \ldots, 4$. Since the relation $R$ is reflexive then we allow that $x_{i}=x_{0}$ for some $i$. We have constructed a model in which one point $x_{0}$ sees at least three others (excluding itself). Hence we get a contradiction with the axiom $\left(a l t_{3}\right)$.

Second, we prove that there is no interpolant for $\alpha \rightarrow \beta$. 
Lemma 2. For the defined above formula $\alpha \rightarrow \beta$ there is no formula $\gamma$ such that $\operatorname{Var}(\gamma) \subset \operatorname{Var}(\alpha) \cap \operatorname{Var}(\beta)=\{p\}, \alpha \rightarrow \gamma \in \mathbf{K T B}$.Alt $(\mathbf{3})$ and $\gamma \rightarrow \beta \in \mathbf{K T B}$.Alt $(3)$.

Proof: Suppose, on the contrary, that there is a formula $\gamma$, written in one variable $p$, such that $\alpha \rightarrow \gamma$ and $\gamma \rightarrow \beta$ are theorems KTB.Alt(3). Then in each reflexive, symmetric and linear Kripke frame $\mathfrak{F}=\langle W, R\rangle$, at any point $x \in W$ and for all valuations $V_{j}$ we get:

$$
x \models_{V_{j}} \alpha \rightarrow \gamma, \quad \text { and } \quad x \models_{V_{j}} \gamma \rightarrow \beta .
$$

Let $\mathfrak{F}=\langle\mathbb{Z}, R\rangle$, where $\mathbb{Z}$ - set of integers, and $R$ is defined in the following way: $n R m$ iff $|n-m| \leq 1$.

Let us consider all valuations $V$ such that $0 \models_{V} \alpha$. Then four possible situations may hold.

1. $V(p) \supset\{-1,0,1\}$ or

2. $V(p) \supset\{-1,0\}$ and $1 \notin V(p)$ or

3. $V(p) \supset\{0,1\}$ and $-1 \notin V(p)$ or

4. $V(p) \supset\{-1,1\}$ and $0 \notin V(p)$.

In all these situations the formula $\gamma$ must be true at the point 0 . We conclude that $0 \models_{V} \gamma$ if $\mathrm{V}$ fulfils one of the conditions (1)-(4). Since $0 \models_{V} \gamma \rightarrow \beta$ then for these valuations $V$ we also get $0 \models_{V} \beta$.

Let us consider the case (4). Without loosing generality we may take $V_{1}$ such that $V_{1}(p)=\{-1,1\}$ and $V_{1}(\alpha)=\{0\}$, and $V_{1}(\gamma) \supset\{0\}$.

On the other side if $\beta$ is false for some valuation then $\gamma$ is false either. $\beta$ may be falsified at the point 0 , for example, for the following valuation $V_{2}: V_{2}(p)=\{-2,1\}$. Then $V_{2}(\gamma) \not \supset\{0\}$.

We restrict ourselves to formulas of one variable $p$. In this way we have defined two different models $\mathfrak{M}_{1}:=\left\langle\mathbb{Z}, R, V_{1}\right\rangle$ and $\mathfrak{M}_{2}:=\left\langle\mathbb{Z}, R, V_{2}\right\rangle$.

There are two different p-morphisms for these models: $f_{1}(k)=|k|$ for all $k \in \mathbb{Z}$ and $f_{2}(-k-1)=f_{2}(k)=k$ for $k \geq 0$. We see that in the images $f_{1}\left(\mathfrak{M}_{1}\right)$ and $f_{2}\left(\mathfrak{M}_{2}\right)$ the valuations $V_{1}$ and $V_{2}$ of variable $p$ will be change as follows: $V_{1}^{*}(p)=\{1\}$ and $V_{2}^{*}(p)=\{1\}$ what means that, in fact, they are identical.

Since p-morphism for models preserves the truth of formulas then we get $V_{1}^{*}(\gamma) \supset\{0\}$ as well as $V_{2}^{*}(\gamma) \not \supset\{0\}$. This is a contradiction. Then the interpolant $\gamma$ for $\alpha \rightarrow \beta$ does not exist. 
From Lemmas 1 and 2 we get

Theorem 5. The logic KTB.Alt(3) does not have (CIP).

One may ask a question if the above counterexample can be applied to show that KTB.Alt(3) does not have (IPD). We shall leave this as an open question.

\section{Interpolation of tabular logics from $N E X T($ KTB.Alt(3))}

It occurred that there is an important connection between (CIP) and Halldén completeness of modal logics. So, we recall definition of the second notion.

Definition 5. A logic $L$ is Halldén complete if

$$
\varphi \vee \psi \in L \text { implies } \varphi \in L \text { or } \psi \in L
$$

for all $\varphi$ and $\psi$ containing no common variables.

Also, we need to recall the definition of the Post completeness for logic. Definition 6. A logic $L$ is said to be Post complete if it is consistent and has no proper consistent extension.

One may notice that each logic from $N E X T(\mathbf{K T B})$ has only one Post complete extension; namely it is the trivial logic Triv $=\mathbf{K} \mathbf{4} \oplus \square p \leftrightarrow p$.

An important connection between (CIP) and Halldén completeness is given by G. F. Schumm in [16] in the following lemma:

Lemma 3. If L has only one Post-complete extension and is Halldén-incomplete, then interpolation fails in $L$.

The above lemma concerns non-normal modal logics. They are logics axiomatized without the rule (RN). Semantically, they are determined by Kripke frames with distinguished points (the so-called unnormal worlds). However we shall consider special kind of Kripke frames in which the choice of distinguished points is completely unimportant. Our key tool to recognize logics with interpolation is a recognition of Halldén complete modal logics and the following lemma due to van Benthem and Humberstone from [1]. 
Lemma 4. If a modal logic $L$ is determined by one Kripke frame, which is homogeneous, then $L$ is Halldén complete.

Definition 7. A Kripke frame $\mathfrak{F}:=\langle W, R\rangle$ is homogeneous if for any $x, y \in W$ there exists an automorphism $f$ of $\langle W, R\rangle$ with $f(x)=y$.

In the paper [11] it is proven that

Theorem 6. Let $\mathfrak{F}:=\langle W, R\rangle$ be KTB-Kripke frame, which is finite and connected. Logic $L(\mathfrak{F})$ is Halldén complete iff the frame $\mathfrak{F}$ is homogeneous.

Defining Halldén complete logics, we are bounded by another theorem due to Lemmon [13]. For non-normal modal logics, the theorem is an equivalence. For normal extensions it has the form of implication only. Following Lemmon we say, that two logics $L_{1}, L_{2} \in N E X T(L)$ are incomparable, if $L_{1} \not \subset L_{2}$ and $L_{2} \not \subset L_{1}$.

Theorem 7. Let $L_{1}, L_{2} \in N E X T(L)$ be two incomparable logics. Then the logic $L_{0}=L_{1} \cap L_{2}$ is Halldén incomplete.

From Theorems 6 and 7 we conclude:

Corollary 1. A Kripke complete and tabular logic from NEXT(KTB), which is Halldén complete must be determined by one connected and homogeneous Kripke frame.

In paper [11] we have described a class of Halldén complete logics within the family of $N E X T(\mathbf{K T B}$.Alt $(\mathbf{3}))$. They are determined by so-called circular frames. Formally, we define:

Definition 8. A circular frame $\mathfrak{C}_{n}:=\left\langle W_{n}, R_{n}\right\rangle, n \geq 3$ is defined as follows:

$$
\begin{aligned}
& W_{n}:=\left\{x_{1}, x_{2}, \ldots, x_{n}\right\}, \\
& R_{n}:=\left\{\left(x_{i}, x_{j}\right), i, j=1,2, \ldots, n,|i-j|[\bmod (n-1)] \leq 1\right\} .
\end{aligned}
$$

We also need a definition of a chain frame.

Definition 9. A chain frame $\mathfrak{C h}_{n}:=\left\langle W_{n}, R_{n}\right\rangle, n \geq 2$ is defined as follows:

$$
\begin{aligned}
& W_{n}:=\left\{x_{1}, x_{2}, \ldots, x_{n}\right\}, \\
& R_{n}:=\left\{\left(x_{i}, x_{j}\right), i, j=1,2, \ldots, n,|i-j| \leq 1\right\} .
\end{aligned}
$$

We also add to the class of chain frames the one point frame $\circ$.

It is easy to notice that circular frames are the only non-trivial homogeneous Kripke frames characterizing logics from $N E X T(\mathbf{K T B} \cdot \mathbf{A l t}(\mathbf{3}))$. 
So, we will study $\operatorname{logics} L\left(\mathfrak{C}_{n}\right), n \geq 3$ as well as two trivial cases $L(\circ)$ and $L(\circ--\circ)$ which are logics determined by one reflexive point or two reflexive points being in symmetric relation, appropriately.

Theorem 8. The logics $L(\circ)$ and $L(\circ-0)$ have (CIP).

Proof. We shall consider amalgamation and superamalgamation properties for frames.

Case 1. Logic $L(\circ)$. The one-element class of frames $\{\circ\}$ after closing under p-morphisms does not change. So, we take as $\mathfrak{F}_{0}, \mathfrak{F}_{1}, \mathfrak{F}_{2}$ and $\mathfrak{F}$, the same frame $\circ$. All the needed p-morphisms are identities. Obviously, (SAPK) also holds.

Case 2. Logic $L(\circ--\circ)$. The one-element class of frames $\{\circ--\circ\}$ after closing under p-morphisms enlarges to $\{0--\circ, \circ\}$. Suppose we choose as $\mathfrak{F}_{0}$ the frame $\circ$, and as $\mathfrak{F}_{1}$ and $\mathfrak{F}_{2}$ twice the frame $\circ--\circ$. Then the p-morphisms $f_{1}$ and $f_{2}$ will glue $\circ--\circ$ onto $\circ$. The needed frame $\mathfrak{F}$ is $\circ--\circ$ and the p-morphisms onto $\mathfrak{F}_{1}$ and $\mathfrak{F}_{2}$ are identities. Also (SAPK) holds. For other choices the proofs are similarly trivial.

All the possible reductions for circular frames are described in [12]. Each circular frame $\mathfrak{C}_{2 n-1}, n \geq 2$ is reducible to some chain frame $\mathfrak{C h}_{n}$. The p-morphism may be described as gluing 'in half' the circle, see [12], Lemma 15. Further, each chain frame $\mathfrak{C h}_{2 n-1}$ is reducible to the chain frame $\mathfrak{C h}_{n}$, again by gluing 'in half'. A chain frame with an even number of points $\mathfrak{C h}_{2 n}$ is reducible to both frames: $\mathfrak{C h}_{n}$ and $\mathfrak{C h}_{n+1}$, see [12], Lemmas 13-14. We may conclude, by superposition of p-morphisms, that eventually each circle frame is reducible to $\circ--\circ$.

Lemma 5. The logic $L\left(\mathfrak{C}_{3}\right)$ does not have (IPD).

Proof. The one-element class of frames $\left\{\mathfrak{C}_{3}\right\}$ after closing under pmorphisms enlarges to $\left\{\mathfrak{C}_{3}, \circ--\circ, \circ\right\}$. We show that the condition (APK) does not hold. We choose as follows: $\mathfrak{F}_{1}:=\mathfrak{C}_{3}$, and $\mathfrak{F}_{2}:=\mathfrak{C}_{3}$, and $\mathfrak{F}_{0}:=$ $\circ--\circ$. We have to call the elements of the considered frames. Hence: $\mathfrak{F}_{1}:=\langle\{a, b, c\} ; R\rangle, \mathfrak{F}_{2}:=\left\langle\left\{a^{\prime}, b^{\prime}, c^{\prime}\right\} ; R\right\rangle$, and $\mathfrak{F}_{0}:=\langle\{\alpha, \beta\} ; R\rangle$. In all cases $R$ is reflexive and symmetric (what, in fact, involves here being an equivalence relation). As a frame $\mathfrak{F}$ we have to choose $\mathfrak{C}_{3}$. Let $\mathfrak{F}:=$ $\langle\{x, y, z\}, R\rangle$. See Figure 2. The p-morphisms $f_{1}$ and $f_{2}$ are defined as follows: $f_{1}(a)=f_{1}(c)=\beta, f_{1}(b)=\alpha$ and $f_{2}\left(a^{\prime}\right)=f_{2}\left(b^{\prime}\right)=\alpha, f_{2}\left(c^{\prime}\right)=\beta$.

There exists only one p-morphism $\mathfrak{F} \rightarrow \mathfrak{F}_{1}$ up to renaming variables. We define $g_{1}$, for example, as follows $g_{1}(x)=a, g_{1}(y)=b, g_{1}(z)=c$. 
Then $x \stackrel{g_{1}}{\longrightarrow} a \stackrel{f_{1}}{\longrightarrow} \beta$. Because only for $c^{\prime}$ we have $c^{\prime} \stackrel{f_{2}}{\longrightarrow} \beta$ then we must define $g_{2}(x)=c^{\prime}$ and we get $\left(f_{1} \circ g_{1}\right)(x)=\left(f_{2} \circ g_{2}\right)(x)$.

So we try to define $g_{2}$ for $z$. We have that $z \stackrel{g_{1}}{\longrightarrow} c \stackrel{f_{1}}{\longrightarrow} \beta$. Because only for one element $c^{\prime}$ we get $f_{2}\left(c^{\prime}\right)=\beta$, then we have to define $g_{2}(z)=c^{\prime}$. But then $g_{2}$ is not a p-morphism. We get a contradiction.

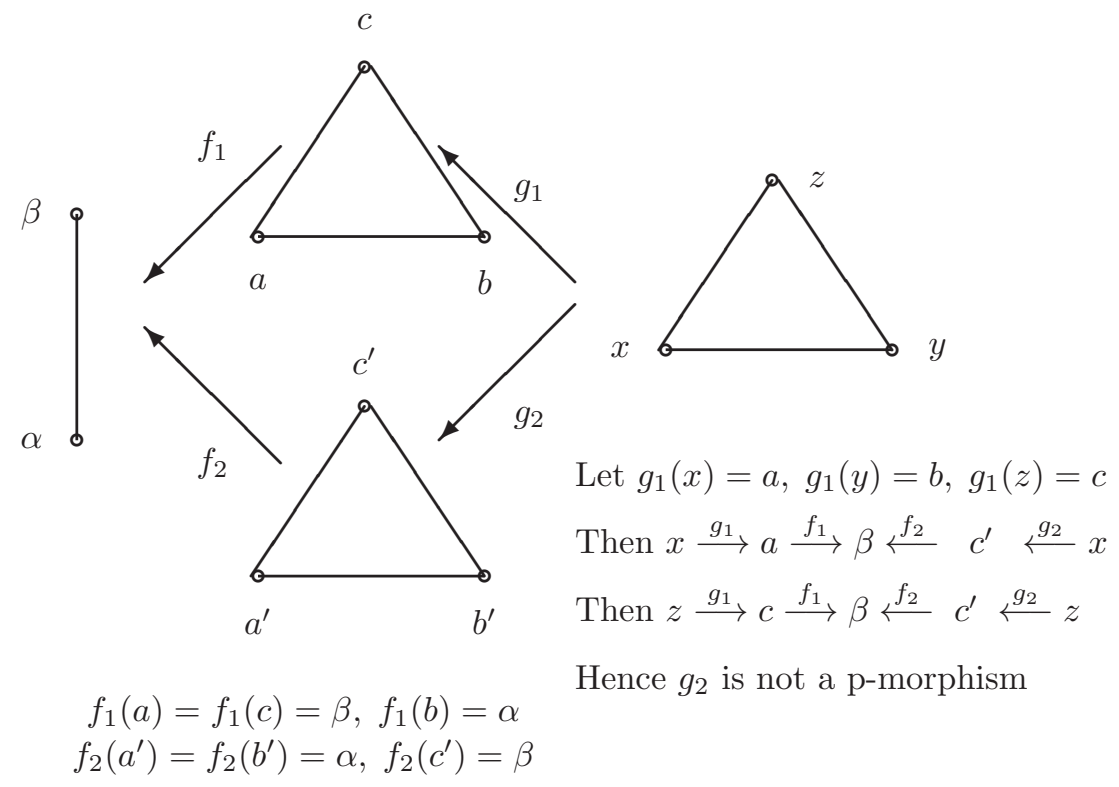

Figure 2 .

We shall similarly prove that

Lemma 6. No logic $L\left(\mathfrak{C}_{2 n-1}\right)$ with $n \geq 3$, has (IPD).

Proof. Instead of making the full proof, we provide it for $n=7$, to avoid a mess with indices.

First, we describe the class of possible reductions of $\mathfrak{C}_{7}$. It is not reducible to any other circle frame, but is reducible to $\mathfrak{C h}_{4}$ and then to $\mathfrak{C h}_{2}:=0--\circ$ and finally to $\circ$. So we chose the needed frames as follows: $\mathfrak{F}_{1}:=\mathfrak{C}_{7}, \mathfrak{F}_{2}:=\mathfrak{C}_{7}$ and $\mathfrak{F}_{0}:=\mathfrak{C h}_{4}$. Let $\mathfrak{F}_{1}:=\left\langle\left\{x_{1}, \ldots, x_{7}\right\} ; R\right\rangle, \mathfrak{F}_{2}:=$ $\left\langle\left\{x_{1}^{\prime}, \ldots, x_{7}^{\prime}\right\} ; R\right\rangle$ and $\mathfrak{F}_{0}:=\langle\{a, b, c, d\} ; R\rangle$. The relation $R$ is reflexive and symmetric. 
We define the p-morphisms $f_{1}: \mathfrak{F}_{1} \rightarrow \mathfrak{F}_{0}$ and $f_{2}: \mathfrak{F}_{2} \rightarrow \mathfrak{F}_{0}$ as follows:

$$
\begin{aligned}
f_{1}\left(x_{1}\right)=d, & f_{2}\left(x_{1}^{\prime}\right)=f_{2}\left(x_{2}^{\prime}\right)=d \\
f_{1}\left(x_{2}\right)=f_{1}\left(x_{7}\right)=c, & f_{2}\left(x_{3}^{\prime}\right)=f_{2}\left(x_{7}^{\prime}\right)=c \\
f_{1}\left(x_{3}\right)=f_{1}\left(x_{6}\right)=b, & f_{2}\left(x_{4}^{\prime}\right)=f_{2}\left(x_{6}^{\prime}\right)=b \\
f_{1}\left(x_{4}\right)=f_{1}\left(x_{5}\right)=a, & f_{2}\left(x_{5}^{\prime}\right)=a
\end{aligned}
$$

Then as the frame $\mathfrak{F}$ we have to take $\mathfrak{C}_{7}$ and the p-morphism $g_{1}: \mathfrak{F} \rightarrow \mathfrak{F}_{1}$ is a unique one up to renaming variables. Let $\mathfrak{F}:=\left\langle\left\{y_{1}, \ldots, y_{7}\right\} ; R\right\rangle$ and $g_{1}\left(y_{i}\right)=x_{i}$ for $i:=1, \ldots, 7$.

Then we get: $y_{4} \stackrel{g_{1}}{\longrightarrow} x_{4} \stackrel{f_{1}}{\longrightarrow} a, x_{5}^{\prime} \stackrel{f_{2}}{\longrightarrow} a$ hence for $g_{2}$ we must take: $g_{2}\left(y_{4}\right)=x_{5}^{\prime}$ and we have $\left(g_{1} \circ f_{1}\right)\left(y_{4}\right)=\left(g_{2} \circ f_{2}\right)\left(y_{4}\right)$. The we try to define in the appropriate way $g_{2}$ for $y_{5}$. We have: $y_{5} \stackrel{g_{1}}{\longrightarrow} x_{5} \stackrel{f_{1}}{\longrightarrow} a$, also only for $x_{5}^{\prime}$ we have $x_{5}^{\prime} \stackrel{f_{2}}{\longrightarrow} a$. So we must take $g_{2}\left(y_{5}\right)=x_{5}^{\prime}$. But then $g_{2}$ is not a p-morphism.

A quite analogous proof may be provided for other odd numbers, with an analogous choice of frames $\mathfrak{F}_{1}:=\mathfrak{C}_{2 n-1}, \mathfrak{F}_{2}:=\mathfrak{C}_{2 n-1}, \mathfrak{F}_{0}:=\mathfrak{C h}_{n}$, with $n \geq 3$.

Lemma 7. No logic $L\left(\mathfrak{C}_{2 n}\right)$ with $n \geq 2$ has (IPD).

Proof. Instead of making the full proof, we provide it for $n=4$, again to avoid a mess with indexes. The one-element class of frames $\left\{\mathfrak{C}_{4}\right\}$ after closing under p-morphisms enlarges to $\left\{\mathfrak{C}_{4}, \mathfrak{C h}_{3}, \circ--\circ, \circ\right\}$. We define a counterexample for (APK). We choose as follows: $\mathfrak{F}_{1}:=\mathfrak{C}_{4}$, and $\mathfrak{F}_{2}:=$ $\mathfrak{C h}_{3}$, and $\mathfrak{F}_{0}:=0--0$. We call the elements of the considered frames. Hence: $\mathfrak{F}_{1}:=\langle\{a, b, c, d\} ; R\rangle, \mathfrak{F}_{2}:=\left\langle\left\{a^{\prime}, b^{\prime}, c^{\prime}\right\} ; R\right\rangle$, and $\mathfrak{F}_{0}:=\langle\{\alpha, \beta\} ; R\rangle$. In all considered cases $R$ is reflexive and symmetric. As a frame $\mathfrak{F}$ we have to choose $\mathfrak{C}_{4}$. Let $\mathfrak{F}:=\langle\{x, y, z, w\} ; R\rangle$. The p-morphisms $f_{1}$ and $f_{2}$ are defined as follows: $f_{1}(a)=f_{1}(d)=\beta, f_{1}(b)=f_{1}(c)=\alpha$ and $f_{2}\left(a^{\prime}\right)=f_{2}\left(c^{\prime}\right)=\alpha, f_{2}\left(b^{\prime}\right)=\beta$. There exists only one p-morphism $\mathfrak{F} \rightarrow \mathfrak{F}_{1}$ up to renaming variables. We define $g_{1}$, for example, as follows $g_{1}(x)=$ $a, g_{1}(y)=b, g_{1}(z)=c$ and $g_{1}(w)=d$. We obtain $x \stackrel{g_{1}}{\longrightarrow} a \stackrel{f_{1}}{\longrightarrow} \beta$. Since $b^{\prime} \stackrel{f_{2}}{\longrightarrow} \beta$ then we must define $g_{2}(x)=b^{\prime}$ and we get $\left(f_{1} \circ g_{1}\right)(x)=\left(f_{2} \circ g_{2}\right)(x)$. Then we consider another element $w$.

We have that $w \stackrel{g_{1}}{\longrightarrow} d \stackrel{f_{1}}{\longrightarrow} \beta$. Because only for one element $b^{\prime}$ we get $f_{2}\left(b^{\prime}\right)=\beta$, then we have to define $g_{2}(w)=b^{\prime}$. But then $g_{2}$ is not a p-morphism. We get a contradiction. See Figure 3. 
A quite analogous proof may be provided for other even numbers, with an analogous choice of frames $\mathfrak{F}_{1}:=\mathfrak{C}_{2 n}, \mathfrak{F}_{2}:=\mathfrak{C h}_{n}, \mathfrak{F}_{0}:=\mathfrak{C h}_{2}$, with $n \geq 3$.

As a conclusion we obtain:

Corollary 2. No tabular logic from NEXT(KTB.Alt(3)) distinct from $L(\circ)$ or $L(\circ--\circ)$ has (IPD) (and (CIP)).

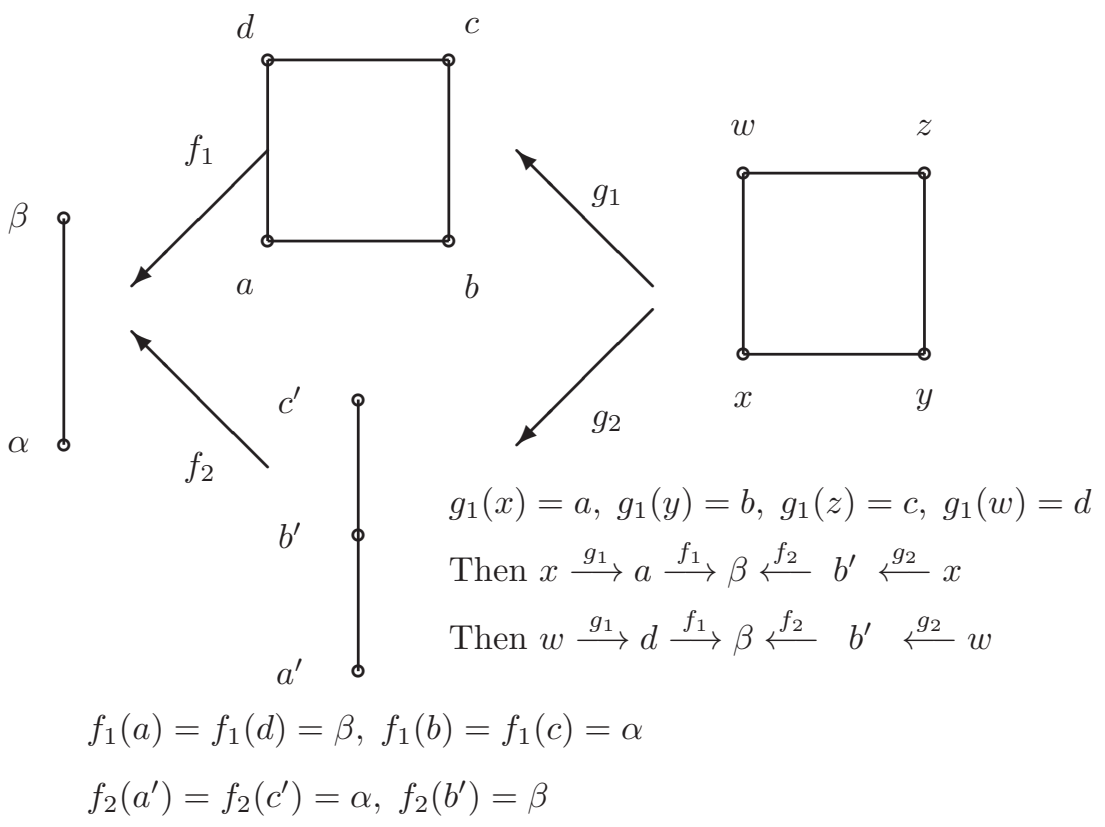

Figure 3.

\section{Problems}

In the paper we prove many negative results on interpolation in the family $N E X T($ KTB.Alt $(\mathbf{3}))$. Our future work will concern interpolation within $N E X T(\operatorname{KTB} \cdot \operatorname{Alt}(\mathbf{n}))$, for $n \geq 4$. For each $n \geq 1$, the logic KTB.Alt(n) is complete with respect to the class of reflexive and symmetric Kripke frames $\mathfrak{F}$ such that each point in $\mathfrak{F}$ sees at most $n$ others (including itself). 
Looking for tabular logics with interpolation among $N E X T($ KTB.Alt(n)) we have to consider homogeneous Kripke frames, that are here, for example, Platonic and Archimedean solids. But not only. The problem is very interesting; in fact describing p-morphisms in such cases in not a trivial job. In some simple cases it seems to be easier. First, we would like to prove that:

Conjecture 1. The logic determined by a reflexive and symmetric Kripke frame having the structure of a Boolean cube has (IDP).

The logic determined by such a cube belongs to KTB.Alt(4). In the area of logics determined by Kripke frames with a larger degree of branching, we also would like to show that

Conjecture 2. The logic determined by a reflexive and symmetric Kripke frame having the structure of $2^{n}$-element Boolean cube, $n \geq 3$, has (IDP).

Acknowledgement. The author is grateful to Piotr Wojtylak, who improved the formula and the proofs in Section 3.

\section{References}

[1] J. F. A. K. van Benthem, I. I. Humberstone, Halldén-completeness by Gluing of Kripke Frames, Notre Dame Journal of Formal Logic 24, No 4 (1983), pp. 426-430.

[2] M. Byrd, D. Ullrich, The extensions of $\mathrm{BAlt}_{3}$, Journal of Philosophical Logic 6 (1977), pp. 109-117.

[3] M. Byrd, The extensions of BAlt ${ }_{3}$-revisited, Journal of Philosophical Logic 7 (1978), pp. 407-413.

[4] A. Chagrov, M. Zakharyaschev, Modal Logic, Oxford Logic Guides 35, (1997).

[5] J. Czelakowski, Logical matrices and the amalgamation property, SL 41 (4), (1981), pp. 329-341.

[6] D. M. Gabbay, Craig's interpolation theorem for modal logics, [in:] W. Hodges, editor, Proceedings of logic conference, London 1970, Vol. 255 of Lecture Notes in Mathematics, Springer-Verlag, Berlin, (1972), pp. 111-127.

[7] S. Halldén, On the semantic non-completeness of certain Lewis calculi, The Journal of Symbolic Logic 16 (1951), pp. 127-129. 
[8] Z. Kostrzycka, On Modal Systems in the Neighbourhood of the Brouwer Logic, Acta Universitatis Wratislaviensis No 3238, Logika 25, Wydawnictwo Uniwersytetu Wrocawskiego, Wrocaw (2010).

[9] Z. Kostrzycka, On interpolation and Halldén-completeness in $N E X T(K T B)$, Bulletin of the Section of Logic 41:1/2 (2012), pp. 23-32.

[10] Z. Kostrzycka, Y. Miyazaki, Normal modal logics determined by aligned clusters, Studia Logica (2017), pp. 105-111.

[11] Z. Kostrzycka, On Hallden completeness of modal logics determined by homogeneous Kripke frames, Bulletin of the Section of Logic 44:3/4 (2015), pp. $1-20$.

[12] Z. Kostrzycka, All splitting logics in the lattice NEXT(KTB.3'), Scientific Issues Jan Długosz University in Częstochowa, Mathematics XXI (2016), pp. 31-61.

[13] E. J. Lemmon, A note on Halldén-incompleteness, Notre Dame Journal of Formal Logic, VII, No 4, (1966), pp. 296-300.

[14] L. Maksimowa, Amalgamation and Interpolation in Normal Modal Logics, Studia Logica, Vol. 50 (3/4), (1991), pp. 457-471.

[15] J. C. C. McKinsey, Systems of modal logics which are not unreasonable in the sense of Hallden, Journal of Symbolic Logic 18 (1953), pp. 109-113.

[16] G. F. Schumm, Some failures of interpolatin in modal logic, Notre Dame Journal of Formal Logic 27 (1), (1986), pp. 108-110.

University of Technology

Sosnkowskiego 31, 45-272, Opole

Poland

e-mail: z.kostrzycka@po.opole.pl 